\title{
A Psychophysical Approach to Dimensional Separability
}

\author{
Patricia W. Cheng and Robert G. PaChella
}

\section{University of Michigan}

\begin{abstract}
Combinations of some physically independent dimensions appear to fuse into a single perceptual attribute, whereas combinations of other dimensions leave the dimensions perceptually distinct. This apparent difference in the perceived distinctiveness of visual dimensions has previously been explained by the postulation of two types of internal representations - integral and separable. It is argued that apparent integrality, as well as its intermediate forms, can result from a single type of representation (the separable type), due to various degrees of correspondence between physical and separable psychological dimensions. Three experiments tested predictions of this new conceptualization of dimensional separability. Experiment 1 demonstrated that a physical dimension corresponding to a separable psychological dimension did not produce interference, whereas a physical dimension not corresponding to a separable psychological dimension did produce interference. Experiment 2 showed that the pattern of results obtained in Experiment 1 could not be accounted for by similarity relations between stimuli. Experiment 3 showed that degrees of correspondence could account for different amounts of interference as well as an inverse relationship between interference and condensation time. These findings imply that previous definitions of integrality are inadequate. Two new converging criteria are proposed, based on the invariance of perceived values on psychological dimensions and on the effect of rotating a configuration of stimuli in a multidimensional space. The present findings furthermore raise the possibility that a single type of internal representation may sufficiently account for all phenomena previously believed to arise from integrality.
\end{abstract}

Certain stimulus dimensions, such as shape and color, are phenomenologically distinct in a compelling fashion; whereas others, such as brightness and saturation, are in combination perceived as a more unitary entity. Some theorists have suggested that younger children perceive holistically, whereas older children learn to differentiate perceptual dimen-

This research was supported by the Office of Naval Research Contract N00014-76-C0648. This paper is based in part on a PhD dissertation completed by Patricia Cheng under the advisorship of Robert Pachella. We thank David Meyer, Wilfred Kincaid, Gary Olson, and members of the ONR research group for their helpful comments, Carol Luckhart for running half of the subjects and analyzing part of the data, and especially Keith Holyoak and Patricia Somers for their generous help. We also thank Naomi Weisstein and three anonymous referees for their helpful comments and suggestions on an earlier draft of this paper. Reprint requests should be addressed to Patricia Cheng, University of Michigan, Human Performance Center, 330 Packard Rd., Ann Arbor, MI 48104. 
sions (Bruner, Olver, \& Greenfield, 1966; E. J. Gibson, 1969). Such apparent differences in the perceived distinctiveness of visual dimensions have provided the impetus for a growing literature on the internal representation of visual information (e.g., Garner, 1974, 1978; Kemler \& Smith, 1979; Lockhead, 1966, 1972; Shepard, 1964; Torgerson, 1958). A number of phenomena have been associated with variations in dimensional distinctiveness. We will briefly review some of these phemomena and discuss a type of theory that has been proposed to account for them. We will then describe an alternative theory and report three experiments designed to test it.

When sets of stimuli that vary orthogonally along some stimulus dimensions, such as the value and chroma of color chips, are classified according to a specified dimension, reaction time (RT) is increased by orthogonal variation along the irrelevant dimension (Garner \& Felfoldy, 1970). In contrast, the analogous task with other pairs of stimulus dimensions, such as the number and shape of objects, is performed without interference from the irrelevant dimension (Fitts \& Biederman, 1965). This task has sometimes been called filtering (Posner, 1964), because of the requirement that the person filter out one dimension while attending to the other.

A second phenomenon concerns facilitation of classification performance when values of stimuli are correlated across dimensions. Whereas correlation facilitates performance for some combinations of stimulus dimensions, such as length and position of a line (Lockhead, 1966), correlation has no effect for other combinations of stimulus dimensions, such as size and saturation of grey squares (Smith \& Kemler, 1978).

A third phenomenon concerns reaction time in what has been called a condensation task (Posner, 1964). This task requires that subjects attend to information on two or more orthogonally varying dimensions. Subjects respond more quickly in a condensation task with dimensions that produce interference in a filtering task than with dimensions that do not produce interference.

These phenomena have been used to infer a distinction between two types of internal perceptual representations. A number of theorists have claimed that objects represented by separable dimensions are perceived in terms of their separate values on these dimensions, whereas objects represented by integral dimensions are not perceived dimensionally at all, but are perceived holistically in terms of their overall similarity (Garner \& Felfoldy, 1970; Lockhead, 1966; Lockhead \& King, 1977). Garner and his co-workers, for example, systematically proposed that the phenomena mentioned serve as part of a set of converging operations for defining integrality and separability: integral dimensions are those that produce interference when sclective attention (filtering) is required, pro- 
duce facilitation with correlated dimensions, and require a relatively short time to condense (summarized in Garner, 1974). In the present paper the term "integrality" will be used to denote a particular type of theory accounting for variations in dimensional distinctiveness. The more neutral term "inseparability" will be used to refer to phenomena relating to the apparent fusing of stimulus dimensions.

As an illustration of the relationship between the above-mentioned phenomena and the two types of internal representations, consider the following. Figure 1 is a schematic diagram of a set of four stimuli generated by an orthogonal combination of values on two dimensions. To measure a subject's ability to selcctively attend to, say, dimension $X$, the time to classify stimuli A and C against B and D (an orthogonal classification) is compared to the average amount of time to discriminate $C$ from $D$ and to discriminate $\mathrm{A}$ from $\mathrm{B}$, each of which is a unidimensional classification. If a person is able to selectively attend to dimension $X$, then the orthogonal classification task should not take any longer than the unidimensional classitication task. Presumably, information about values on dimension $Y$ can be ignored, so that $\mathrm{A}$ and $\mathrm{C}$ are psychologically the same stimulus (since they share the same value on dimension $X$ ), as are $B$ and $D$. In such a case, classifying four stimuli varying independently on separable dimensions should take no longer than classifying two. In contrast, similarity relations are assumed to be important with integral stimuli. Since there is dissimilarity (i.e., a nonzero Euclidean distance) between $A$ and $C$, and likewise between $B$ and $D$, each of the response classes has two distinct stimuli, as compared to only one for separable dimensions. This within-class variability will increase classification time so that classifying four distinct stimuli will take longer than classifying two. The mean increase in classification time from the unidimensional to

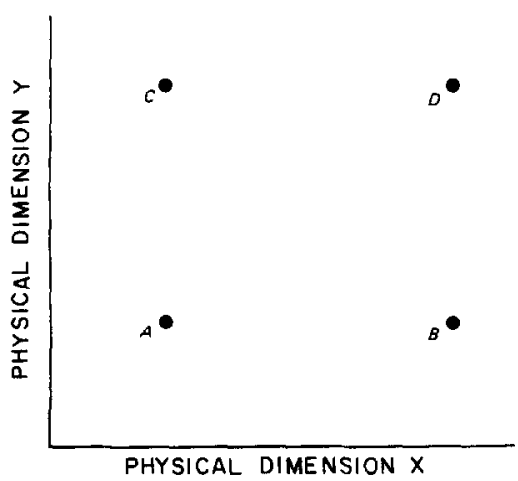

FIG. 1. A schematic diagram of four stimuli generated by orthogonal variation on two physical dimensions. Dots represent stimuli. 
the orthogonal classification task is referred to as interference. For integral stimuli, the irrelevant dimension (dimension $Y$ in this example) interferes with classification on the basis of the relevant dimension (dimension $X$ ). The more dissimilar the stimuli are within a response class, the greater the expected interference; and the more dissimilar the stimuli are between the response classes, the less the expected interference (Garner, 1974; Lockhead \& King, 1977).

Some difficulties with Garner's (1974) definition of integrality have been discussed in Pachella, Somers, and Hardzinski (1981). One major problem is that the putative converging operations have frequently failed to converge. For example, Garner and Felfoldy (1970) found that dimensions that were compellingly inseparable yielded the expected facilitation of reaction time with correlated dimensions, but surprisingly little interference when selective attention was required. This led Garner (1974) to suggest that integrality might be a continuum, thus allowing degrees of inseparability. In a similar vein, Smith and Kemler (1978) proposed a "continuum of dimensional primacy." The concept of degrees of integrality is quite incongruous with the concept of two distinct types of psychological structures, and the nature of potential intermediate structures has not been specified.

A further complication was introduced by Pomerantz and Sager (1975). After concluding that two dimensions were integral on the basis of interference on both dimensions, they went on to call the dimensions "asymmetrically integral" because the amounts of interference on the two dimensions were unequal. Kemler and Smith (1979) found that subjects had a tendency to use dimensional rather than similarity relations in a concept learning task, even for the ostensibly inseparable dimensions of brightness and saturation. Such intermediate combinations of results have led to a burgeoning taxonomy of types of integrality (Garner, 1974, 1978; Garner \& Felfoldy, 1970; Kemler \& Smith, 1979; Pomerantz \& Sager, 1975; Smith \& Kemler, 1978), with seemingly little explanatory power.

\section{Psychophysical Correspondence}

In view of the growing chaos surrounding the concept of integrality, we propose an alternative approach. Our approach is psychophysical, in the sense that it attempts to find simple, functional relationships between physical and psychological variables.

Our theory begins with the observation that there are infinitely many possible physical descriptions of a stimulus. As William James noted,

out of the infinite chaos of movements, of which physics teaches us that the outer world consists, . . out of what is in itself an undistinguishable, swarming continuum, devoid of distinction or emphasis, our senses make for us, by attending 
to this motion and ignoring that, a world full of contrasts, of sharp accents, of abrupt changes, of picturesque light and shade. (1890, pp. 284-285)

Out of this infinite number of possible physical descriptions, people choose a finite number to encode the stimulus psychologically. We propose that physical dimensions that correspond to these psychological attributes are separable, whereas as those that do not correspond to psychological attributes are inseparable. A psychological attribute is defined as onc that can be selectively attended to, in the sense that a perceived value on such an attribute remains constant when the value on the corresponding physical dimension remains constant, despite variation on irrelevant physical dimensions.

Consider, for instance, the description of a parallelogram. A parallelogram can be specified by any three orthogonal parameters, such as the lengths of two adjacent sides and an angle, the lengths of one side and one diagonal and the height, the lengths of two adjacent sides and one diagonal, and so on. For simplicity of exposition let us vary only two dimensions by fixing the third, say the ratio of the lengths of adjacent sides. Suppose it were the case that out of the various possible physical dimensions that can describe a parallelogram under the above restriction, only two dimensions - any one of the angles and the length of the longer diagonal-corresponded to the psychological attributes of shape and size. These attributes, by definition of being psychological, could be attended to selectively. That is, if one of these, say angle, were kept constant, perceived shape should remain constant despite variation on other dimensions. The left panel of Fig. 2 depicts four parallelograms varying orthogonally along angle and length of a side. As can be seen, despite variation on the length of the sides, parallelograms with the same angles appear the same shape. Consequently, when no extraneous sources of interference are present, classification according to angle should yield no interference.

In contrast, when the physical dimension manipulated does not correspond to any psychological attribute, perception will vary even though the physical dimension is being kept constant. To illustrate the point, let us now fix the length of the horizontal sides so that shape, the hypothetical psychological dimension, can vary more freely. Consider keeping the height (hypothetically a nonpsychological dimension) constant while varying the length of the oblique sides. Parallelograms with a given height can have many shapes and sizes (hypothetically psychological dimensions), depending on the length of the oblique sides. The right panel of Fig. 2 depicts parallelograms varying orthogonally along height and length of the oblique sides (with length of the horizontal sides fixed). As can be seen, parallelograms of the same height do not appear obviously identical along any compelling attribute. They vary in shape and size despite the 


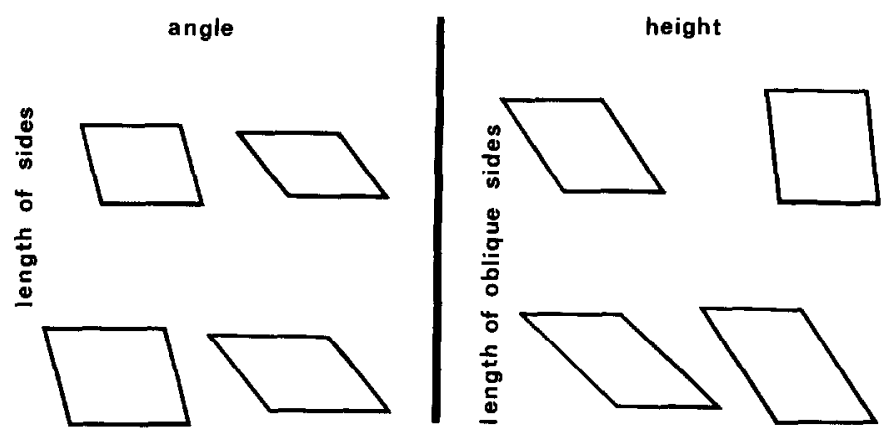

Fig. 2. Left panel: Parallclograms varying orthogonally on angle and length of side (with ratio of lengths of adjacent sides kept constant). Right panel: Parallelograms varying orthogonally on height and length of oblique sides (with length of horizontal sides kept constant).

constant heights. Since values on the psychological dimensions vary despite the constancy of the nonpsychological dimension, classification according to a nonpsychological dimension should yield interference due to this variability. Incidentally, note that although parallelograms of the same height in the figure have the same area, they do not appear the same size, illustrating that psychological attributes may not have obvious physical correlates.

To put our approach in perspective, we should mention that J. J. Gibson $(1960,1966)$ proposed a similar approach to the classical problem of perceptual constancies. He proposed that the perplexing lack of correlation between proximal stimulation and perception may well be due to the arbitrary physical dimensions that have been chosen to describe the proximal stimulus. According to this view, our percepts may be direct functions of some higher ordered, physical variables. If only we could find the appropriate physical variables, a psychophysics of perception may exist. Analogously, we suggest that if only we could find the appropriate separable dimensions, performance might be a direct function of relations on these dimensions. As a result, there may be no need to postulate a multitude of types of integrality, or even integrality itself.

In the first experiment below we will show that psychological dimensions produce no interference in orthogonal classification, whereas nonpsychological dimensions do produce interference. In a subsequent experiment we will argue that psychophysical mapping can also account for degrees of inseparability, and the inverse relationship between interference and condensation time.

\section{EXPERIMENT 1}

Experiment 1 was designed to demonstrate that whereas physical di- 
mensions corresponding to psychological attributes will not produce interference, physical dimensions not corresponding to psychological attributes will. To accomplish this we chose different dimensions describing the same stimuli. In this and subsequent experiments our stimuli will be triangles. Like any other stimuli, triangles may be physically specified any number of ways: by the lengths of three sides, by the lengths of two sides and an included angle, by the size of two angles and an included side, and so on. One of the dimensions chosen in the present experiment is psychological, whereas another is nonpsychological. Following our definition in the Introduction, we assumed that a physical dimension corresponds to a psychological attribute if and only if the perceived value of a stimulus on this dimension remains compellingly unchanged when other physical dimensions are varied. Our strategy was to first demonstrate the validity of our theory with obviously psychological and obviously nonpsychological dimensions, before buttressing our theory with a converging definition that is supported by empirical results to be described in Experiment 3. We varied the psychological and nonpsychological dimensions independently with an irrelevant dimension and measured how quickly and accurately subjects classified stimuli using each of the relevant dimensions. To assess the effect of the irrelevant dimension, we also measured how quickly and accurately subjects classified stimuli when there was no variation on the irrelevant dimension. Our prediction was that stimuli classified according to a psychological dimension would not produce interference, whereas stimuli classified according to a nonpsychological dimension would produce interference. Unless one is willing to argue that the domain of stimuli used in these experiments (triangles) is represented internally in two qualitatively different ways, such a pattern of results cannot be explained by current theories of integrality.

Since the physical dimensions examined in our experiments were chosen to be either compellingly psychological or compellingly nonpsychological, perceptions of such dimensions should be stable across different individuals. Accordingly, each experiment involves a detailed examination of the performance of a small number of subjects, and the data were analyzed by individual subjects.

Although Experiment 1 was run as part of Experiment 2, for clarity of exposition they are reported as separate experiments.

\section{Method}

Subjects. The subjects were four paid, undergraduate students at the University of Michigan. All had normal vision. None had any previous experience with the tasks used in this study.

Stimuli. Two pairs of sets, A and B, were constructed. Each pair of sets comprised a 
psychological and nonpsychological set. Each set consisted of four right-leaning isosceles triangles with a horizontal base. The lengths of the base and the right side were equal. The triangles were presented on a cathode ray tube (CRT) controlled by a PDP-1 computer. They subtended approximately 6.3 to $11.5^{\circ}$ of visual angle horizontally and 2.2 to $5.7^{\circ}$ vertically.

The triangles in each set varied orthogonally on two dimensions. One of the dimensions in each set, designated the relevant dimension, determined class membership in the speeded classification task. The other dimension in each set was irrelevant for speeded classification. The irrelevant dimension was common across all sets.

For psychological set $\mathrm{A}$ the relevant dimension was shape, a compelling psychological dimension. This set is displayed in Fig. 3, in which the dotted line denotes the required partition into categories. As can be seen, despite variation on the length of the right side (the irrelevant dimension), triangles in the same category still appear obviously identical in shape. Levels of right-side length were $22 / 16$ and $25 / 16$ in. Levels of shape are denoted by the exterior angle at the right of the base (as it will continue to be throughout this paper); the values were 39 and $61^{\circ}$.

In contrast, for nonpsychological set $\mathrm{A}$, the relevant dimension was not compellingly psychological. As can be seen in Fig. 4, triangles in the same category do not appear obviously identical along any compelling dimension. For this set the relevant dimension was height; levels of height were $14 / 16$ and $22 / 16$ in. The irrelevant dimension was the length of the right side, as in the psychological set. Levels of right-side length were the same as those in the psychological set.

For the second pair of sets the irrelevant dimension for both sets was again the length of right side. The relevant dimension for psychological set $B$ (Fig. 5) remained shape, but the relevant dimension for nonpsychological set $B$ (Fig. 6) was changed to length of left side. Levels of shape were 53 and $70^{\circ}$ for the psychological set. Levels of left-side length were $36 / 16$ and $43 / 16$ in. for the nonpsychological set. The levels of right-side length for both sets were $22 / 16$ and $24 / 16$ in.

To avoid confounding due to interclass distance, the minimum interclass distance along the relevant psychological dimension was kept constant for each pair of sets. This minimum distance was the distance between the two levels of the psychological dimension for each pair of sets. This control measure maintained a constant minimum interclass distance across psychological and nonpsychological sets, and kept average interclass distances shorter for

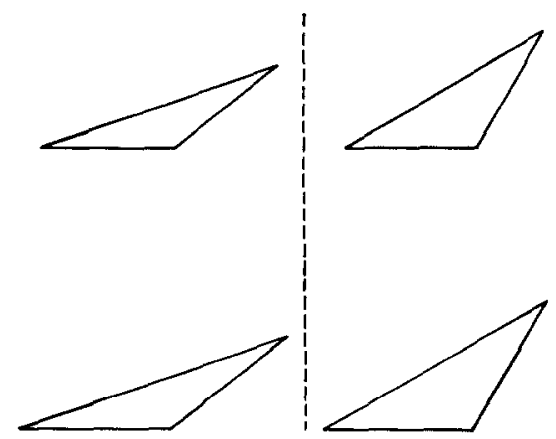

FIG. 3. Experiment 1: Psychological set A, formed by orthogonal variation on the shape and the length of the right side of isosceles triangles. The dashed line denotes the required partition. 

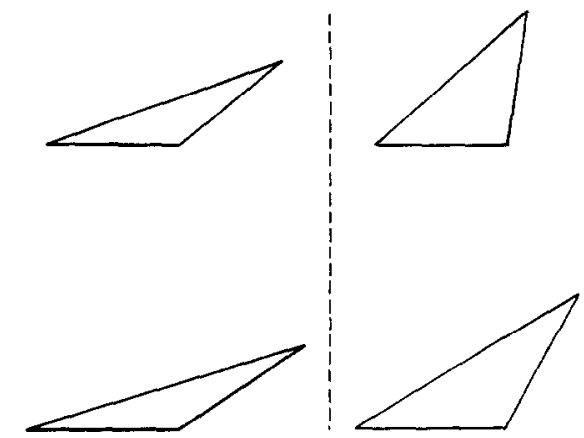

FIG. 4. Experiment 1: Nonpsychological set A, formed by orthogonal variation on the height and the length of the right side of isosceles triangles.

psychological than nonpsychological sets. Consequently, a small amount of interference obtained for the psychological sets cannot be attributable to interclass distance, since greater interclass similarity should yield greater interference.

Procedure. Two subjects received the A sets and two received the B sets. The procedure was the same for both sets for every subject. Triangles were presented one at a time on the center of a CRT screen. Subjects were required to classify each into one of two categories by pressing with their right or left index finger on one of two microswitches on a panel. Before each task, instructions for classifying a particular set of triangles were presented on the CRT screen. These instructions displayed one triangle of the set at a time, accompanied by the word "left" or "right," corresponding, respectively, to the response categories of pressing the left or right response key. Subjects were instructed to respond as quickly as possible while keeping error rate below $3 \%$.

Each subject participated in a practice session followed by a test session. Practice sessions were identical to test sessions. Each session consisted of two blocks. Each block included one run of orthogonal and of unidimensional classification tasks on a psychological and a nonpsychological set. The classification tasks were randomly ordered within a block. A run for each task on a session consisted of 12 practice trials followed by 40 actual trials.
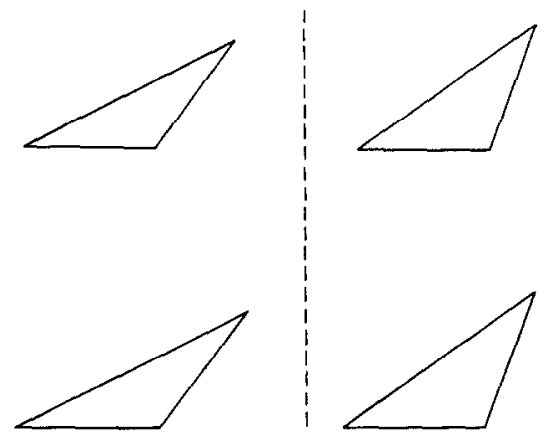

FIG. 5. Experiment 1: Psychological set B, formed by orthogonal variation on the shape and the length of the right side of isosceles triangles. 

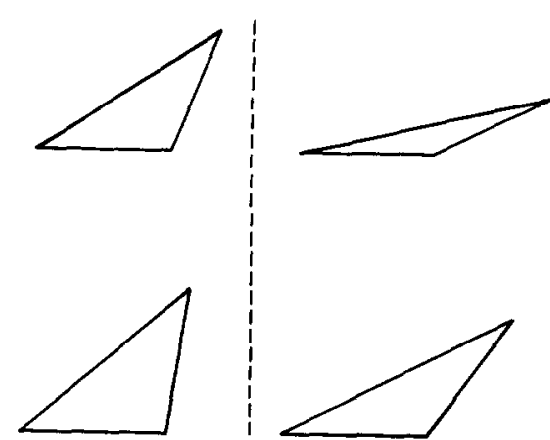

FIG. 6. Experiment 1: Nonpsychological set $B$, formed by orthogonal variation on the length of the left side and the length of the right side of isosceles triangles.

For sets with two triangles, there were 20 actual presentations of each triangle. For sets with four triangles, there were only 10 presentations of each, in order to keep the length of each task constant. Each session lasted from 40 to 60 min.

\section{Results and Discussion}

Mean error rates were below $4 \%$ for all subjects. Correlations between $\mathrm{RT}$ for correct responses and for error responses across blocks were high, .70 or above $(p<.05)$ for all subjects. To simplify data analyses RTs for error trials were included.

We predicted that when the relevant dimension was psychological, orthogonal variation on the irrelevant dimension would not produce perceptual variability within a response category, and consequently would not produce interference. In contrast, when the relevant dimension was not psychological, orthogonal variation on the irrelevant dimension should produce psychological variability in the response category, and consequently should produce interference. The mean RTs in various conditions are listed in Table 1. Amount of interference is defined as the difference between the mean RTs for the orthogonal and the unidimensional tasks. Error margins reported are $95 \%$ confidence intervals. An analysis of variance was run for each individual subject, with the factors of Correspondence (psychological versus nonpsychological), Task (orthogonal versus unidimensional), Run, and Trial (nested within the above factors). The main effect of the factor Trial was used as the error term for testing contrasts.

Results for the A sets were as predicted by psychophysical correspondence. The amount of interference for the psychological dimension was small and statistically insignificant, $2 \pm 19 \mathrm{~ms}$ for Subject 1 and $-10 \pm$ $18 \mathrm{~ms}$ for Subject 2, $p>.25$ for each subject. (A minus sign indicates a difference reflecting longer RT for unidimensional than for orthogonal 
TABLE 1

Mean RTs (milliseconds) for Orthogonal and Unidimensional Classification for Stimulus Sets with Psychological and Nonpsychological Dimensions (Experiment 1)

\begin{tabular}{lccccc}
\hline & \multicolumn{2}{c}{ A sets } & \multicolumn{2}{c}{ B sets } \\
\cline { 2 - 3 } \cline { 5 - 6 } & Psychological & Nonpsychological & Psychological & Nonpsychological \\
\hline Orthogonal & 338 & 372 & 393 & 404 \\
Unidimensional & 342 & 326 & 385 & 348 \\
Interference & -4 & 46 & 8 & 52 \\
\hline
\end{tabular}

classification.) In contrast, the amount of interference for the nonpsychological dimension was relatively large and statistically significant, 31 $\pm 19 \mathrm{~ms}$ for Subject 1 and $62 \pm 18 \mathrm{~ms}$ for Subject $2 ; p<.001$, for each subject. The difference in interference between these two conditions was $29 \pm 28 \mathrm{~ms}(p<.05)$ for Subject 1 and $72 \pm 26 \mathrm{~ms}(p<.001)$ for Subject 2 .

The B sets used length of left side instead of height as the relevant nonpsychological dimension. The third subject's results followed the same pattern as that obtained with the A sets. The amount of interference for the psychological dimension, $-8 \pm 27 \mathrm{~ms}$ was small and statistically insignificant $(p>.25)$. In contrast, the amount of interference for the nonpsychological dimension, $87 \pm 27 \mathrm{~ms}$ was relatively large and statistically significant $(p<.001)$. The difference in interference between these two conditions was $95 \pm 38 \mathrm{~ms}(p<.001)$. Subject 4 produced a statistically insignificant amount of interference in both the psychological and the nonpsychological sets ( $24 \pm 29 \mathrm{~ms}$ for both sets, $p>.10$ ).

In sum, the results were consistent with our hypothesis: physical dimensions corresponding to psychological dimensions did not produce interference, while physical dimensions not corresponding to psychological ones did produce interference. In other words, our results demonstrated that interference, a phenomenon previously attributed to integrality, may be due to lack of correspondence.

Although we propose that lack of correspondence is a source of interference, we do not imply that it is the only source. Distraction, response conflict, and even integrality, for example, are all possible alternative sources. Thus the presence of interference in itself does not necessarily imply a lack of correspondence. Only when all sources are absent would no interference result. When sources other than lack of correspondence are present across conditions involving both psychological and nonpsychological dimensions, correspondence theory would predict a greater amount of interference for nonpsychological than psychological dimensions. Degrees of interference will be discussed further in Experiment 3. 


\section{EXPERIMENT 2}

A proponent of integrality theory might argue that the pattern of results obtained in Experiment 1 was due to differences in the discriminability between stimuli, rather than differences in the psychophysical correspondence of the stimulus dimensions. Triangles in the same category appear more similar overall in the psychological sets (Fig. 3 and 5) than in the nonpsychological sets (Fig. 4 and 6). It might therefore be argued that all the dimensions manipulated in Experiment 1 were integral and that the apparent absence of interference for the psychological dimension was due to the relatively small degree of dissimilarity that the irrelevant dimension generated between stimuli in the same category. Such an explanation would in fact follow from the model of integrality proposed by Lockhead and King (1977; Monahan \& Lockhead, 1977). This model distinguishes between two types of representations (integral and separable), but postulates that performance on integral dimensions is predicted not by differences on physical dimensions, but by psychological overall similarity, as measured by Euclidean distances in a multidimensional configuration of similarity judgments. In particular, this model predicts that interference would depend on such similarity relations. In contrast, our correspondence hypothesis postulates only one type of representation and attempts to explain performance by psychological similarity as measured by distances along separable psychological dimensions. Although overall similarity has been shown to predict performance for "integral" dimensions, similarity along psychological dimensions was not controlled in such studies (Lockhead \& King, 1977; Monahan \& Lockhead, 1977); overall similarity may therefore have been confounded with similarity along psychological dimensions.

To test Lockhead and King's (1977) hypothesis against our correspondence hypothesis, we varied the overall similarity between stimuli within the same category in such a way that values along the psychological dimension were kept constant. If the internal representation is indeed integral, a decrease in overall within-category similarity should increase interference. However, if similarity along the psychological dimension (i.e., psychophysical correspondence) is what predicts performance, then a decrease in overall within-category similarity per se should not affect performance.

\section{Method}

To the stimulus sets used in Experiment 1 we added sets of triangles that matched the previous sets on values along the psychological dimension, but were less similar along the irrelevant dimension. Similarity judgments on pairs of triangles were collected to confirm this decrease in overall similarity, and classification speeds on orthogonal and unidimensional classification were measured. 
Subjects. The four subjects tested in Experiment 1 also served in Experiment 2.

Stimuli. In addition to the two sets of triangles in stimulus set A of Experiment 1, we constructed two other sets of triangles that matched the previous sets on values along shape, the relevant and hypothetically psychological dimension. Within each set there were two levels of the irrelevant dimension, length of right side. The two triangles with the shorter right side were the same as those in the previous sets, while the two with the longer right side had a longer right side than those in the previous sets. The levels of right-side length were $22 / 16$ and $28 / 16$ in. as compared to $22 / 16$ and $25 / 16$ in. for the two previous sets. Analogously, in addition to the two sets of triangles in stimulus set B of Experiment 1, we constructed two other sets of triangles. The levels on right side were 22/16 and 29/16 in. as compared to $22 / 16$ and $24 / 16$ in. for the previous sets.

Procedure. In the dissimilarity judgment task subjects were instructed to rate the overall dissimilarity of pairs of triangles. After initial familiarization with the triangles in the set (presented one at a time in random order), the subject was presented with each of the possible pairs of triangles, presented one pair at a time in random order. The subject, by pushing 1 of 10 microswitches numbered from 1 to 10 from left to right on a panel, rated the dissimilarity of each pair. The task was self-paced. At the end of each block there was a rest period of about 2 min. The number of blocks within each session varied between six and eight. Each session lasted about $50 \mathrm{~min}$. A practice session was followed by two test sessions.

In addition to judging dissimilarity, subjects classified sets of triangles in the same manner as in Experiment 1. They classified sets of four triangles with right side as the irrelevant dimension. They also classified the triangles unidimensionally to provide the baseline for assessing interference. The classification tasks in this experiment were randomly interspersed with those in Experiment 1.

\section{Results and Discussion}

According to Lockhead and King's model, greater within-category overall dissimilarity should lead to greater interference. In contrast, according to our theory of psychophysical correspondence, discriminability along the psychological dimension should predict interference. Since discriminability along the psychological dimension was kept constant across sets with varying overall dissimilarity, it follows that there should be no difference in interference across these sets.

Within-category dissimilarities. To assess the effect of within-category overall dissimilarity, such variability must first be measured. Accordingly, the mean dissimilarity ratings for stimuli pairs for each subject were submitted to multidimensional scaling, using the nonmetric multidimensional scaling program CONSCAL written by Noma and Johnson (1977). The distance between a pair of stimuli in the best two-dimensional Euclidian configuration was taken as the measure of dissimilarity between the pair.

The two-dimensional scaling solution for one of the subjects was degenerate, as groups of stimuli were collapsed into a few points. ${ }^{1}$ For the

${ }^{1}$ For this subject mean dissimilarity ratings for the relevant pairs were used as a measure of dissimilarity. The resulting ratios confirmed the pattern obtained for the other subjects using scaling solutions. 
other three subjects, stress values were $.05, .08$, and .16 , respectively. For these subjects, the mean of the ratio of within-category dissimilarity for sets with large variation on the irrelevant dimension to within-category dissimilarity for sets with small variation was 1.78 for stimulus set $A$ and 1.97 for stimulus set $B$. In comparison, the mean of the ratio of intracategory distances for nonpsychological sets to those for psychological sets was 1.43 for stimulus set $A$ and 2.19 for stimulus set $B$. These results confirm that, for the sets used in Experiment 2, the manipulations of psychophysical correspondence and of variability on the irrelevant dimension produced comparable differences in overall similarity.

Speeded classification. Mean error rates for speeded classification were below $4 \%$ for all subjects. Correlations between RTs for correct responses and for error resopnses across blocks were again high, .70 or above $(p<.05)$ for all subjects.

An analysis of variance was run for each subject separately, with the factors of Correspondence, Within-category dissimilarity, Task, Run, and Trial (nested within the above factors). The main effect of the factor Trial was used as the error term for testing contrasts. Error margins reported from here on are $95 \%$ confidence intervals calculated by the Scheffe procedure. The mean RTs for orthogonal and unidimensional classification for the two pairs of sets are shown in Table 2 . The results were as predicted by psychophysical correspondence-changes in overall withincategory dissimilarity had no effect on the amount of interference for any of the subjects. For stimulus set A, the difference between interference produced by sets with large and small within-category dissimilarity was $22 \pm 29 \mathrm{~ms}(p>.10)$ for Subject 1 , and $-1 \pm 26 \mathrm{~ms}(p>.25)$ for Subject 2. The comparable difference for stimulus set B was $-21 \pm 41$ ms $(p>.25)$ for Subject 3, and $14 \pm 38 \mathrm{~ms}(p>.25)$ for Subject 4. In contrast, besides producing little or no interference, psychological sets produced significantly less interference than nonpsychological sets for most of the subjects, confirming our results in Experiment 1. For stimulus set $\mathbf{A}$, the difference between interference produced by psychological and nonpsychological sets was $30 \pm 29 \mathrm{~ms}(p<.05)$ for Subject 1 and $41 \pm$ $26 \mathrm{~ms}(p<.001)$ for Subject 2 . For stimulus set B, the corresponding difference was $89 \pm 41 \mathrm{~ms}(p<.001)$ for Subject 3 , and $-11 \pm 38 \mathrm{~ms}$ $(p>.25)$ for Subject 4 .

In sum, changes in overall within-category similarity per se had no effect on the amount of interference for any of our subjects, but nonpsychological dimensions did produce more interference than psychological dimensions for most of our subjects. This pattern of results argues against Lockhead and King's hypothesis that overall similiarity can account for differences in interference, and supports our interpretation in terms of psychophysical correspondence. 
TABLE 2

Means RTs (milliseconds) for Orthogonal and Unidimensional Classification for Each of the Stimulus Sets (Experiment 2)

\begin{tabular}{|c|c|c|c|c|}
\hline Task & $\begin{array}{l}\text { Within-category } \\
\text { dissimilarity }\end{array}$ & Psychological & Nonpsychological & $\bar{x}$ \\
\hline \multicolumn{5}{|c|}{ A sets } \\
\hline \multirow[t]{3}{*}{ Orthogonal } & Large & 361 & 368 & 365 \\
\hline & Small & 338 & 372 & 355 \\
\hline & $\bar{x}$ & 350 & 370 & \\
\hline \multirow[t]{3}{*}{ Unidimensional } & Large & 339 & 326 & 333 \\
\hline & Small & 342 & 326 & 334 \\
\hline & $\bar{x}$ & 341 & 326 & \\
\hline \multicolumn{5}{|c|}{ B sets } \\
\hline \multirow[t]{3}{*}{ Orthogonal } & Large & 405 & 394 & 399 \\
\hline & Small & 393 & 404 & 398 \\
\hline & $\bar{x}$ & 399 & 399 & \\
\hline \multirow[t]{3}{*}{ Unidimensional } & Large & 393 & 351 & 372 \\
\hline & Small & 385 & 348 & 366 \\
\hline & $\bar{x}$ & 389 & 349 & \\
\hline
\end{tabular}

Smith (1981) found that adults as well as children structured their free classifications of complex objects (i.e., objects that varied simultaneously on relatively many dimensions) on the basis of overall-similarity relations, rather than identity on compelling psychological dimensions such as shape and color. As Smith herself pointed out, however, this finding does not imply that separable dimensions become more integral as more dimensions vary. First, the claim that objects are apprehended as wholes does not necessarily imply the holistic processing of component parts. Subjects may have processed each dimension of difference separately but based their decisions about classification on the combination of these independent differences. Smith's finding that older subjects were generally able to classify the complex sets by identity along at least one component dimension provides some empirical support for this view. Moreover, Strutt, Anderson, and Well (1975) found no effect of number of varying irrelevant dimensions on adults' performance in a speeded selective-attention task, indicating that the ability to selectively attend to a separable dimension is independent of stimulus complexity.

\section{EXPERIMENT 3}

Gottwald and Garner (1975) proposed that whereas stimuli varying on integral dimensions produce interference in a filtering task and those 


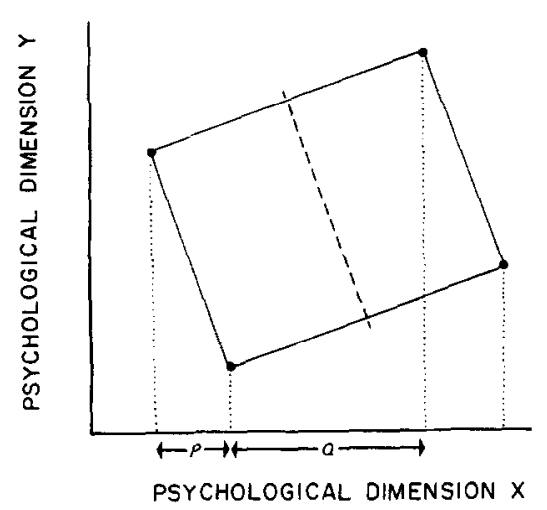

FIG. 7. An illustration explaining an inverse relationship between amount of interference and RT for diagonal classification. The dashed line denotes the required partition; the dotted lines denote projections onto the $x$ axis.

varying on separable dimensions do not, stimuli varying on integral dimensions will be classified more quickly than those varying on separable dimensions in a condensation task (which requires that the subject pay attention to two or more dimensions). This prediction follows from the assumption that subjects classify stimuli varying on integral dimensions on the basis of similarity relations, and hence to not have to attend separately to two dimensions in turn, as they do for separable dimensions.

However, the inverse relationship between interference in a filtering task and classification time in a condensation task could also result from the correspondence of physical dimensions and psychological attributes. The simplest condensation task, used in Experiment 3, requires that the subject classify the diagonals of an orthogonal set of stimuli into two categories (i.e., A and D versus $B$ and $C$ in Fig. 1). This task will be termed diagonal classification. Figure 7 illustrates how degrees of correspondence can result in an inverse relationship between reaction time for diagonal classification and interference. Referring to the figure, for orthogonal classification the distance $P$ is a measure of the variability within a response category, and the distance $Q$ is a measure of the variability between categories. Conversely, for diagonal classification $Q$ indicates the variability within one of the response categories, and $P$ the variability between categories. Thus orthogonal classification should be easier as $P$ becomes smaller and $Q$ becomes larger. The opposite is true for diagonal classification. Now, consider rotations of the stimulus set from the orientation where $P$ equals zero to that where $Q$ equals zero. As $P$ increases, $Q$ decreases. Thus as orthogonal classification becomes harder, diagonal classification becomes easier. Consequently, an inverse relationship between interference and diagonal classification time can result from degrees of correspondence, which can be caused by rotations 
of a configuration of stimuli relative to definable psychological attributes, Indeed, differing degrees of correspondence can lead to apparent "degrees of integrality." The purpose of Experiment 3 was to demonstrate that this inverse relationship between interference and diagonal classification time can indeed result from the correspondence or the lack of it in the mapping between physical and psychological dimensions.

\section{Method}

To show an inverse relationship between interference and RT on diagonal classification, subjects performed speeded classification tasks for stimulus sets for which two-dimensional scaling configurations were rotated relative to each other. Subjects performed orthogonal, diagonal, and unidimensional classification on stimulus sets of comparable discriminability and configuration (both sets had roughly rectangular scaling configurations). In Part A subjects classified stimulus sets at each orientation according to one dimension, which at one of the orientations appeared to correspond to perceived size. The psychophysical correspondence theory predicts that stimulus sets aligned with a psychological dimension will produce no interference but relatively long RTs for diagonal classification, whereas stimulus sets oriented at an angle to the psychological dimension will produce interference but relatively short RTs for diagonal classification.

Part B was analogous to Part A except that subjects classified stimuli according to both dimensions at both orientations, none of which appeared to correspond to any psychological attribute. Psychophysical correspondence predicts that stimulus sets of both orientations should produce interference (since none of the dimensions correspond to any psychological attribute), but sets with an orientation more closely aligned with a psychological dimension should produce less interference and a longer reaction time on diagonal classification. This predicted pattern of results is intermediate between the patterns used by Garner to define integral and separable dimensions, and hence would demonstrate that degree of psychophysical correspondence can produce apparent "degrees of integrality."

Subjects. Subjects were four undergraduates at the University of Michigan, who were paid for their participation in the experiment. Two served in Part A and two in Part B. All had normal vision. None had any prior experience with tasks in this study.

Stimuli. For each subject the stimuli were two sets of triangles, eight in each sel, with some common members. Stimulus sets were developed individually for each subject. This was done in order to keep scaling configuration similar across the two orientations for each subject. Pilot work showed that scaling configurations of the same set of stimuli varied irregularly across subjects. ${ }^{2}$

Subjects judged the dissimilarity of all possible pairs of triangles in their stimulus sets. The procedure for this task was the same as in Experiment 2. Each session lasted about $50 \mathrm{~min}$. Due to individual differences in the speed of making dissimilarity judgments, the number of blocks within each session varied between four and seven across subjects. The number of dissimilarity judgment sessions for each subject varied from five to nine.

The two-dimensional configurations derived from the dissimilarity judgments of each subject for each set were roughly rectangular, and the axes of each set were oriented at an angle to each other. The configuration of the lowest stress for 16 stimuli for one of the subjects appears in Fig. 8. The configurations for other subjects are similar. The minimum stress values were .05 and .06 for the subjects in Part A, and .09 and .05 for the subjects in Part B. Referring to Fig. 8, solid lines connect stimuli in the "original" set; dashed lines

\footnotetext{
${ }^{2}$ There was no need to develop individual sets of stimuli in Experiments 1 and 2 because the ordering of the critical variables (dissimilarity along a psychological dimension and overall dissimilarity) was constant across subjects for a single set of stimuli.
} 


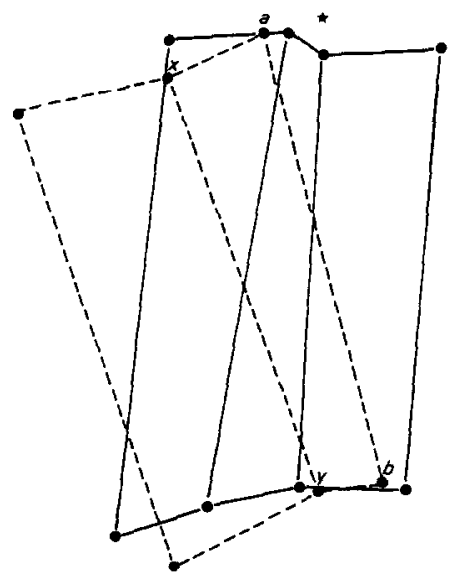

FIG. 8. Two-dimensional scaling configuration derived from dissimilarity ratings on $\mathbf{1 6}$ triangles for one subject in Part A of Experiment 3. Dots represent stimuli included in speeded classification; stars represent stimuli excluded from speeded classification.

connect those in the "rotated" set. There were always three sets of four triangles each for orientation, but not all triangles appearing in the dissimilarity judgment task appeared in the speeded classification tasks; only those that formed rectangular configurations were used. For example, for the stimulus set based on the configuration depicted in Fig. 8, the stimuli chosen were connected into rectangular groups in the figure. For the subjects in Part A, sets were constructed such that discriminability along the relevant dimension for the rotated set was greater than or equal to that for the original set, and discriminability along the irrelevant dimension for the rotated set was less than or equal to that for the original set. ${ }^{3}$ These criteria ensured that greater interference for the rotated set could not be attributed to either its lesser discriminability along the relevant dimension or its greater discriminability along the irrelevlant dimension.

Figure 9 shows the original and rotated sets of stimuli in Part A for the subject whose scaling configuration appears in Fig. 8. As can be seen, for the original set the horizontal axis of the scaling configuration corresponds to perceived size, and the vertical axis corresponds to perceived shape. The same correspondence held for the other subject in Part A.

Classification procedure. The procedure was similar across the two parts. Subjects classified six rectangular subsets of stimuli in the same manner as in Experiment 1 . In addition to the orthogonal and unidimensional tasks, subjects made classifications according to the diagonals of the rectangular subsets of stimuli. For the orthogonal and unidimensional tasks, they classified stimuli according to the horizontal dimension for the original set. The horizontal dimension was length of right side, the dimension that appears to correspond closely to perceived size for the subjects in Part A. For the rotated set, these subjects classified according to the dimension for which discriminability between stimuli was most similar to discriminability along the horizontal dimension of the original set. For example, for the stimulus set corresponding to the scaling configuration depicted in Fig. 8, stimuli a and $b$ were classified against stimuli $x$ and $y$.

${ }^{3}$ It was not possible to control relative discriminability in this manner for the stimulus sets used in Part B, since classifications were performed according to both dimensions at each orient: tion. 


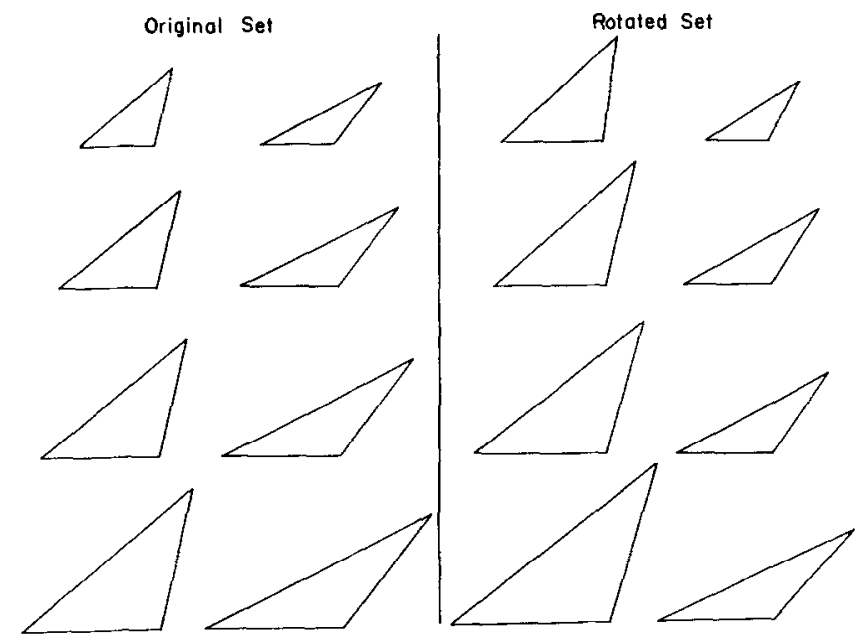

FIG. 9. Triangles in the original and rotated sets of Experiment 3.

In Part B none of the dimensions corresponded exactly with any psychological dimension. Subjects classified stimuli by both dimensions for each orientation. We predicted that sets with an orientation more closely aligned with a psychological dimension would produce less interference and longer diagonal classification time.

Each subject performed two runs of each classification task for each orientation. Each run for each orientation took from $45 \mathrm{~min}$ to an hour. The ordering of orientations was counterbalanced across runs. Each subject participated in four sessions, each of which lasted from 45 min to an hour.

\section{Results and Discussion}

Results were analyzed separately for each subject, since each received different stimulus configurations. Error rates for all subjects were $4 \%$ or below. Correlations between the RTs of correct responses and of error responses across blocks were again high, ranging from .66 to .85 ( $p<$ .01). An analysis of variance was run for each subject separately, with the factors of Orientation, Task Type (orthogonal, unidimensional, or diagonal classification), Run, Stimulus Group (the particular group of two or four stimuli in each task, nested within Task Type), and Trial (nested within Stimulus Group). The mean classification times for orthogonal, unidimensional, and diagonal classification for each subject are listed separately in Table 3. The critical contrasts and their $95 \%$ Scheffe confidence intervals are also listed in the table. The main effect of the factor Trial, pooled across tasks, was used as the error term for testing contrasts.

For both subjects in Part A, results were as predicted by psychophysical correspondence. The original set (classified according to length of right side) produced no interference (mean interference was $-4 \mathrm{~ms}, p>$ .25 , for each subject), whereas the rotated set produced a significant 
TABLE 3

Interference and Mean RTs (milliseconds) for Orthogonal, Unidimensional, and Diagonal Classification Tasks for Individual Subjects in Experiment 3, Parts A and B

\begin{tabular}{|c|c|c|c|c|}
\hline & Orthogonal & Unidimensional & Interference & Diagonal \\
\hline & & Original set & & \\
\hline \multicolumn{5}{|l|}{ Part A } \\
\hline Subject 1 & 409 & 419 & $-10 \pm 35$ & 617 \\
\hline Subject 2 & 333 & 331 & $2 \pm 25$ & 620 \\
\hline \multicolumn{5}{|l|}{ Part B } \\
\hline \multicolumn{5}{|l|}{ Subject 3} \\
\hline Dimension 1 & 407 & 369 & $38 \pm 15$ & \multirow{2}{*}{650} \\
\hline Dimension 2 & 426 & 366 & $60 \pm 16$ & \\
\hline \multicolumn{5}{|l|}{ Subject 4} \\
\hline Dimension 1 & 448 & 378 & $70 \pm 17$ & \multirow{2}{*}{761} \\
\hline Dimension 2 & 505 & 479 & $26 \pm 23$ & \\
\hline & & Rotated set & & \\
\hline \multicolumn{5}{|l|}{ Part A } \\
\hline Subject 1 & 470 & 431 & $39 \pm 35$ & 550 \\
\hline Subject 2 & 580 & 339 & $241 \pm 25$ & 458 \\
\hline \multicolumn{5}{|l|}{ Part B } \\
\hline \multicolumn{5}{|l|}{ Subject 3} \\
\hline Dimension 1 & 448 & 380 & $68 \pm 15$ & \multirow{2}{*}{598} \\
\hline Dimension 2 & 473 & 370 & $103 \pm 16$ & \\
\hline \multicolumn{5}{|l|}{ Subject 4} \\
\hline Dimension 1 & 450 & 377 & $73 \pm 17$ & \multirow{2}{*}{648} \\
\hline Dimension 2 & 579 & 479 & $100 \pm 23$ & \\
\hline
\end{tabular}

Note. Error margins are $95 \%$ Scheffe confidence intervals.

amount of interference (mean interference was $140 \mathrm{~ms}, p<.05$, for each subject). The difference was significant for each subject. Diagonal classification time was significantly faster for the rotated set than for the original set. The mean difference in RT was $115 \mathrm{~ms}, p<.01$, for each subjcct.

The results for both subjects in Part B were also in accord with the pattern predicted by psychophysical correspondence. Both the original and rotated sets produced significant interference, but the amounts of interference were significantly greater for the rotated than for the original orientation. The mean difference was $37 \mathrm{~ms}, p<.05$ for each subject. As predicted, diagonal classification time was significantly faster for the orientation with more interference. The mean difference in RT was 63 $\mathrm{ms}, p<.01$, for each subject.

To examine the possibility of confounding due to discriminability for the subjects in Part B (see Footnote 3), we examined the correlation between interference and the relative discriminability of the sets of 
stimuli. Relative discriminability was defined as the ratio of discriminability along relevant versus irrelevant dimensions, where discriminability was indexed by the distance between stimuli in the two-dimensional scaling configuration. The more dissimilar a set of stimuli are along the relevant dimension, relative to their dissimilarity along the irrelevant dimension, the higher the relative discriminability. Higher relative discriminability might be expected to lead to less interference. The correlations between interference and the relative discriminability of the stimulus sets for the two orientations were -.45 and -.43 , respectively, for the two subjects $(p>.10)$. Although insignificant, both correlations were negative, as would be expected if discriminability was important. However, a closer examination of the relationship between relative discriminability and interference indicated that at least for one subject relative discriminability could not account for the pattern of interference. For Subject 3, the relative discriminability for classification by the vertical axis was higher for the rotated set (.65) than for the original set (.55); nonetheless, the rotated set produced more interference. This is contrary to the prediction based on relative discriminability. Thus at least for this subject the results are sufficient ro rule out relative discriminability as an explanation for greater interference for the rotated set.

The results of Experiment 3 indicate that a dimension corresponding to a psychological attribute produces no interference and a relatively long diagonal classification time, whereas dimensions not corresponding to psychological attributes produce interference and a shorter diagonal classification time. Furthermore, the results of Part B indicate that apparent "degrees of integrality" can result from degrees of correspondence between physical and psychological dimensions: the greater the correspondence, the smaller the interference, and the longer the RT for diagonal classification.

\section{GENERAL DISCUSSION}

Experiment 1 demonstrated that classification according to a psychological dimension produced no interference, whereas classification according to a nonpsychological dimension did produce interference. Experiment 2 verified that overall similarity cannot account for the pattern of interference obtained in Experiment 1. Experiment 3 demonstrated that a psychological dimension produced no interference as well as a relatively long RT in condensation, whereas a nonpsychological dimension produced interference as well as a relatively short RT in condensation. Experiment 3 also demonstrated that apparent "degrees of integrality" could arise from differing degrees of correspondence between physical and psychological dimensions. 
We have so far applied our theory to only parallelograms and triangles, and have empirically tested our theory on only the latter. How generalizable is our theory? Consider, for example, Felfoldy's (1974) experiment in which he asked subjects to classify rectangles according to their height or width. He concluded that the dimensions were integral on the basis of interference and redundancy gain. Lockhead and King (1977) manipulated the lengths of two vertical lines, and found that classification performance was not correlated with values along these two physical dimensions. Dixon and Just (1978) varied the height and width of ellipses as well as the hue and tint of color patches in a "same-different" task, and found increasing interference with increasing disparity between stimuli on the irrelevant dimension. Could psychophysical correspondence account for the inseparability observed in such studies? First, note that in the above studies only onc set of dimensions was examined for each set of stimuli. Other plausible dimensions such as the shape and size of rectangles or ellipses, or the ratio of the lengths of vertical lines, were not considered. No justifications were offered for the apparently arbitrary choices. Second, results from a study in which alternative dimensions of rectangles were tested indeed suggested that the shape of a rectangle, rather than its height or width, is psychological. Using an absolute judgment task, Weintraub (1971) measured information transmission on various subsets of 15 rectangles selected from a total set formed from the combination of 15 heights and 15 widths. Some of these subsets varied on single dimensions that were oriented at $45^{\circ}$ to each other. The mean information transmission for subsets that varied on either height or width (rows or columns from the matrix of $15 \times 15$ ) was significantly less than that for the subset that varied along the diagonal of the matrix. The diagonal consisted of 15 rectangles of approximately equal area but varying in shape. These results corroborate our results for triangles, and provide further support for the relevance of psychophysical correspondence in the interpretation of inseparability.

Psychophysical correspondence can account not only for interference, condensation time, and degrees of inseparability, but also for other phenomena associated with integrality, such as redundancy gain, free classification performance, and asymmetric separability. Let us consider each of these phenomena in turn. According to Garner and Felfoldy (1970), integral dimensions yield shorter classification time (i.e., redundancy gain) when the dimensions are correlated. However, redundancy gain could arise from a lack of psychophysical correspondence. Somers (1978) showed that stimuli correlated on nonpsychological dimensions can sometimes be more discriminable than univariate stimuli, and that such correlated stimuli produce redundancy gain. In addition, she showed that 
depending on the particular mapping of physical and psychological dimensions, stimuli correlated on nonpsychological dimensions can sometimes be less discriminable than univariate stimuli. Such correlated stimuli should and indeed yield redundancy loss, a result which cannot be explained by integrality.

Another phenomenon often associated with integrality is performance in free classification. Whereas stimuli varying on integral dimensions are grouped according to overall similarity relations (maximizing withingroup similarity and minimizing between-group similarity), stimuli varying on separable dimensions are grouped according to a shared value on one dimension, ignoring overall similarity relations and values on the second dimension (Handel \& Imai, 1972). Such free classification results can also be explained by psychophysical correspondence. Stimuli varying on psychological dimensions can be grouped according to a shared value on one dimension. However, stimuli varying on nonpsychological dimensions will not have a shared value psychologically. Without a basis for grouping according to a dimension, subjects may resort to grouping by overall similarity.

Previous discussions of integrality and separability have invariably defined these terms as attributes of pairs of dimensions, since integrality and separability have been assumed to arise from ways in which physical dimensions combine with each other. Some combinations remain distinct, while others interact to form a new, integral psychological dimension. In contrast, the correspondence theory provides an alternate framework for analyzing perceptual dimensions. According to this theory, it is not the combination per se, but the lack of correspondence between physical and psychological dimensions which leads to phenomena associated with integrality. It follows that the separability of individual dimensions can be evaluated alone, without reference to any particular second dimension.

This inherent feature of our theory provides a ready explanation of "asymmetric integrality." This term has been applied to cases in which the amounts of interference on two orthogonal dimensions are unequalcases in which one of two orthogonally varied dimensions produces interference whereas the other does not (Garner, 1974, referring to Day \& Wood, 1972, and Wood, 1974), and cases in which one of the dimensions produces greater interference than the other (Pomerantz \& Sager, 1975). Apparent "asymmetric integrality" is not readily explicable by Garner's theory of two types of internal representations. To explain the asymmetry, various strategies and levels of processing have been hypothesized (Garner, 1974; Pomerantz \& Sager, 1975). Within the alternative framework of psychophysical correspondence, the same phenomenon might more naturally be labeled "asymmetric separability": if one dimension 
is psychological and another is not, the latter dimension will produce interference whereas the former will not.

It thus appears that psychophysical correspondence can account for the patterns of performance previously used to define integrality; moreover, it can account for intermediate combinations of results not explicable by such definitions. Psychophysical correspondence has implications for integrality on two levels of generality. Within the framework of two distinct types of representations, it implies that previously proposed operations do not critically differentiate between integral and separable dimensions. Accordingly, results based on these operations are uninterpretable. In place of these operations, we propose testing for the constant perception of a given value on a physical dimension despite variation on irrelevant physical dimensions (as discussed in the Introduction), and for the effect of the rotation of axes (as in Experiment 3). Whereas classification performance for integral dimensions should never be affected by the rotation of axes, that for separable dimensions should be affected by rotations that create different degrees of correspondence (as was the case in Experiment 3).

Since none of the previously proposed operations can identify integral dimensions, what evidence is there then to indicate that integral dimensions exist at all? Such evidence so far is ambiguous. One study that examined the effect of the rotation of axes suggests that integral dimensions may indeed exist. Smith and Kemler (1978) reported that in general the rotation of axes did not affect classification performance for the dimensions of brightness and saturation, indicating that these dimensions are integral. However, rotation of axes did have a significant effect on classification time for some of the stimulus sets (Smith \& Kemler, 1978, Experiment 3). Moreover, these investigators also reported in another study (Kemler \& Smith, 1979) that subjects in general have a preference for dimensional relations in a concept learning task involving brightness and saturation, indicating that these dimensions are separable. The evidence that color dimensions are integral thus appears ambiguous; outside of the color domain there seems at present to be no evidence that requires postulation of integral dimensions. More broadly, then, our results raise the possiblity that integral dimensions may be a myth: lack of correspondence may account for all phenomena previously believed to arise from integrality.

\section{REFERENCES}

Bruner, J. S., Olver, R. R., \& Greenfield, P. M. (1966). Studies in cognitive growth. New York: Wiley. 
Day, R. S., \& Wood, C. C. (1972). Interactions between linguistic and nonlinguistic processing. Journal of the Acoustical Society of America, 51, 79.

Dixon, P., \& Just, M. A. (1978). Normalization of irrelevant dimensions in stimulus comparisons. Jounral of Experimental Psychology: Human Perception and Performance, 4, 36-46.

Egeth, H., \& Pachella, R. G. (1969). Multidimensional stimulus identification. Perception \& Psychophysics, 5, 341-346.

Felfoldy, G. L. (1974). Repetition effects in choice reaction time to multidimensional stimuli. Perception \& Psychophysics, 15, 453-459.

Fitts, P. M., \& Biederman, I. (1965). S-R compatibility and information reduction. Journal of Experimental Psychology, 69, 408-412.

Garner, W. R. (1974). The processing of information and structure. Potomac, MD: Erlbaum.

Garner, W. R. (1978). Selective attention to attribute and to stimuli. Journal of Experimental Psychology: General, 107, 287-308.

Garner, W. R., \& Felfoldy, G. L. (1970). Integrality of stimulus dimensions in various types of information processing. Cognitive Psychology, 1, 225-241.

Gibson, E. J. (1969). Principles of perceptual learning and development. New York: Appleton-Century-Crofts.

Gibson, J. J. (1960). The concept of the stimulus in psychology. American Psychologist, $15,694-703$.

Gibson, J. J. (1966). The senses considered as perceptual systems. Boston: Houghton Mifflin.

Gottwald, R. L, \& Garner, W. R. (1975). Filtering and condensation tasks with integral and separable dimensions. Perception \& Psychophysics, 18, 26-28.

Handel, S., \& Imai, S. (1972). The free classification of analyzable and unanalyzable stimuli. Perception \& Psychophysics, 12, $108-116$.

James, W. (1890). The principles of psychology. Holt.

Kemler, D. G., \& Smith, L. B. (1979). Accessing similarity and dimensional relations: Effects of integrality and separability on the discovery of complex concepts. Journal of Experimental Psychology: General, 108, 133-150.

Lockhead, G. R. (1966). Effects of dimensional redundancy on visual discrimination. Journal of Experimental Psychology, 72, 95-104.

Lockhead, G. R. (1972). Processing dimensional stimuli: A note. Psychological Review, $79,410-419$.

Lockhead, G. R., \& King, M. C. (1977). Classifying integral stimuli. Journal of Experimental Psychology: Human Perception and Performance. 3, 436-443.

Monahan, J. S., \& Lockhead, G. R. (1977). Identification of integral stimuli. Journal of Experimental Psychology: General, 106, 94-110.

Noma, E., \& Johnson, J. (1977, August). Confirmatory multidimensional scaling (Tech. Rep. No. 60). Human Performance Center, University of Michigan.

Pachella, R. G., Somers, P., \& Hardzinski, M. (1981). A psychophysical approach to dimensional integrality. In D. Getty \& J. Howard (Eds.), Auditory and visual pattern recognition. Hillsdale, NJ: Erlbaum.

Pomerantz, J. R., \& Garner, W. R. (1973). Stimulus configuration in selective attention tasks. Perception \& Psychophysics, 14, 565-569.

Pomerantz, J. R., \& Sager, L. C. (1975). Asymmetric integrality with dimensions of visual pattern. Perception \& Psychophysics, 18, 460-466.

Posner, M. I. (1964). Information reduction in the analysis of sequential tasks. Psychological Review, 71, 491-504.

Shepard, R. N. (1964). Attention and the metric structure of the stimulus space. Journal of Mathematical Psychology, 1, 54-87. 
Smith, L. B. (1981). Importance of the overall similarity of objects for adults' and children's classifications. Journal of Experimental Psychology: Human Perception and Performance, 7, 811-824.

Smith, P., \& Kemler, D. G. (1978). Levels of experienced dimensionality in children and adults. Cognitive Psychology, 10, 502-532.

Somers, P. (1978). Perceptual interaction between stimulus dimensions as the basis of dimensional integrality. $\mathrm{PhD}$ dissertation, University of Michigan.

Strutt, G. F., Anderson, D. R., \& Well, A. D. (1975). A developmental study of the effects of irrelevant information on speeded classification. Journal of Experimental Child $\boldsymbol{P}_{\boldsymbol{s} y-}$ chology, 20, 127-135.

Torgerson, W. S. (1958). Theory and methods of scaling. New York: Wiley.

Weintraub, D. J. (1971). Rectangle discriminability: Perceptual relativity and the law of Pragnanz. Journal of Experimental Psychology, 88, 1-11.

Wood, C. C. (1974). Parallel processing of auditory and phonetic information in speech discrimination. Perception \& Psychophysics, 15, 501-508.

(Accepted February 28, 1984) 